Int. J. Electrochem. Sci., 13 (2018) 1905 - 1920

\title{
Differential Pulse Voltammetry Determination of Anti- Hypertensive Drug Hydrochlorothiazide in Pharmaceuticals Using Glassy-Carbon Electrode Modified by Electropolymerization with L- and D- Glutamic Acids
}

\author{
Camilo González-Vargas ${ }^{1}$, Camilo Garcia ${ }^{2}$, Freddy $_{\text {Celis }}{ }^{3}$, Ricardo Salazar $^{1, *}$ \\ ${ }^{1}$ Laboratorio de electroquímica del Medio Ambiente, Departamento de Química de los Materiales, \\ Facultad de Química y Biología, Universidad de Santiago de Chile, USACh, Casilla 40, Correo 33, \\ Santiago, Chile. \\ ${ }^{2}$ Facultad de Recursos Naturales, Escuela de Ciencias Ambientales. Universidad Católica de Temuco. \\ Temuco, Chile. \\ ${ }^{3}$ Centro de estudios avanzados y Departamento de química, Facultad de Ciencias Naturales y Exactas, \\ Universidad de Playa Ancha, Calle Traslaviña 450, Viña del Mar, Chile \\ *E-mail: ricardo.salazar@usach.cl
}

doi: $10.20964 / 2018.02 .10$

Received: 26 June 2017 / Accepted: 24 November 2017 / Published: 28 December 2017

\begin{abstract}
A simple and sensitive electrochemical sensor based on glassy carbon electrode modified by electropolymerization with both poly-glutamic acid enantiomers L- and D (GC-PGA) was developed to detect the anti-hypertensive drug hydrochlorotiazide (6-chloro-3,4-di-hydro-2H-1, 2,4-benzothiadiazine-7-sulfonamide-1,1 dioxide, HCTZ) in pharmaceuticals samples. Raman spectroscopy and electrochemical impedance spectroscopy were carried out to characterize L-PGA and D-PGA film. These procedures confirm the production of a polymer through an amide bond and the formation of a film resistant to charge transference in both cases. Also, an increase in the oxidation peak current for HCTZ in Buffer Britton Robinson solution 0,1 M pH 2 was obtained using the modified electrodes. With GC/D-PGA and GC/L-PGA, the peak current increased 2 times compared to unmodified GC, when using differential pulse voltammetry. A novel electroanalytical method for the determination of HCTZ was developed with both modified electrodes, showing a less limit of determination: 0.03186 and $0.01829 \mathrm{mM}$ to GC/L-PGA and GC/D-PGA, respectively, and a less limit of quantification with respect to the GC electrode. The methodology developed was applied in the determination of HCTZ from pharmaceutical forms.
\end{abstract}

Keywords: Glutamic Acid, Hydrochlorotiazide, electropolymerization, differential pulse voltammetry, glassy carbon electrode. 
(C) 2018 The Authors. Published by ESG (www.electrochemsci.org). This article is an open access article distributed under the terms and conditions of the Creative Commons Attribution license (http://creativecommons.org/licenses/by/4.0/). 